\title{
Size Functions in Galaxy Morphology Classification
}

\author{
Sreeparna Banerjee \\ Department of Natural Science \\ West Bengal University of Technology \\ Kolkata 700064 \\ india
}

\begin{abstract}
This paper introduces the concept of size functions in galaxy morphology classification, which are geometrical-topological shape descriptors which can be tailor-made to describe the shapes of objects of interest. For the three main classes of galaxies obtained from the Hubble classification scheme, namely, elliptical, spiral and lenticular, the size functions are obtained from two sets of measuring functions based on shape and pixel intensities. For elliptical galaxies, the ellipticity is considered for the shape measuring function whereas for spiral galaxies the pitch angle at turnover for the spiral arms is used. For lenticular galaxies the shape is obtained from eccentricity considerations. The intensity measuring functions can be obtained after color segmentation using an Otsu based method. Use of size functions is useful for automating the galaxy classification, as the methodology is robust and allows flexibility. Initial results are promising.
\end{abstract}

\section{General Terms}

Pattern Recognition, Astronomy.

\section{Keywords}

Size functions, galaxy, elliptical, spiral, lenticular, segmentation, Hubble telescope.

\section{INTRODUCTION}

Galaxies are celestial bodies which are composed of gas, dust, and billions of stars bound by gravity. Morphology concerns the the shape and visual appearance of galaxies, giving crucial insight into their composition and evolution. Thus, morphological galaxy classification is important for the explanation of the physical processes governing galaxies, star formation, and the nature of the universe.

In this note, efforts in morphological galaxy classification using a special class of shape descriptors called size functions are described. To this end, existing galaxy classification schemes are presented in the next section followed by allied work in this area. Then the concept of size functions is introduced and definitions for size functions of galaxies in the context of this work are elucidated. Finally some representative examples of size functions for some of the galaxies are given to give an indication of the efficacy of the method.

\section{CLASSIFICATION OF GALAXIES}

Morphological classification of galaxies has been first performed systematically by Hubble [1] and later on efforts by de Vaucouleurs [2] and Sandage [3] have been notable. Hubble's classification scheme, which forms the basis of all classification schemes is based entirely on the shape of the galaxies. According to this scheme, galaxies can be classified as elliptical, spiral, lenticular or irregular. As the name suggest, elliptical galaxies are elliptical in shape and are composed mostly of stars in an advanced stage of their evolution. Spiral galaxies are spiral in shape, usually bi symmetric and with spiraling arms. lenticular galaxies are considered as spiral but without spiraling arms. Irregular galaxies do not exhibit any regular structure. Spiral galaxies with prominent bi symmetric arms are referred to as grand design galaxies and constitute about $10 \%$ of the observed galaxy population. Arms of the multi-arm galaxies are not so prominent but they constitute the the majority of the observed galaxy population $(\sim 60 \%)$. The rest of the observed galaxies whose arms are even less prominent than multi-arm are the flocculent galaxies comprising of $20 \%$ of the observed population.

In addition to shape, the Radial Brightness Profile (RBF) [4] is another important consideration for morphological galaxy classification. Galaxies are composed of stars in various stages of evolution, and the shape of a galaxy is determined by the composition of its stars. For instance, elliptical galaxies are old galaxies composed primarily of stars that have reached their final stage of evolution whereas for spiral and lenticular galaxies, there are regions of new star formation. Regions of older stars comprise the bulge of the star and are reddish in color, while regions of new star formation are the disks, bars and arms, and are bluish in color.

From studies of images of galaxies in five different photometric bands, inferences of the morphological nature of the galaxies can be made. Previous research indicates a good correlation between the Hubble classification and some parameters obtained from the RBF $[5,6]$. These include the disk-to-bulge ratio (DB) and the concentration index (ci).

In order to obtain the signatures necessary for galaxy classification, the 2-D image is de-projected to face-onorientation. Two angles, namely the position angle $(\alpha)$ and the inclination angle $(\beta)$ are required to project the galaxy shape from the "sky plane" to the "image plane". A third angle $(\gamma)$ is required for correct orientation. These three angles constitute the Euler angles $(\alpha, \beta, \gamma)$.

Due to the large numbers of galaxy images that are becoming available in data sets, for example the Sloan Digital Sky Survey (SDSS), it is necessary to automate the morphological classification process. In this note, efforts in this direction. are described Some notable Artificial Intelligence (AI) based approaches to automating galaxy classification include Naive Bayes' classifier, rule-induction algorithm $\mathrm{C} 4.5$ and random forest [7].

\section{SIZE FUNCTIONS}

Size functions are special class of geometry- topology based shape descriptors which are integer valued [8,9]. The idea underlying the concept of size functions is that of setting of metric bounds to the classical notion of homotopy, i.e. continuous deformation. Representation of the size function can be tailored to suit the quantitative and qualitative invariant 
properties of the shape to be studied. The goal of the theory is to produce a representation of the shape of a planar curve. The theory is based on two fundamental concepts, namely, a real valued function defined along the curve or region, called the measuring function and an integer valued function of two real variables called the size function. The size function encodes information about the shape of the curve by looking at the behavior of the measuring function along the curve itself. The size function has a number of interesting properties namely:

1. It can be used to distinguish the shape of topologically equivalent curves.

2. It is robust against small continuous changes of the underlying curve.

3. It can be used to establish a distance between shapes.

4. It can be computed from a distinct approximation of a curve.

5. It can be generalized from curves to surfaces of arbitrary dimension.

\section{SIZE FUNCTIONS FOR GALAXIES}

In this paper two sets of measuring functions (m.f.) are proposed :

One set of measuring functions corresponds to the shape based on Hubble's classification scheme. For elliptical galaxies this measuring function $(\eta)$ is an integer representing the ratio of the major axis (a) to minor axis (b) of the galaxy's isophote, i.e.

$$
\eta=10(1-\mathrm{b} / \mathrm{a})
$$

The classification ranges from E0, which corresponds to a perfectly spherical shape, to E7 in this scheme. lenticular galaxies are considered as spiral galaxies with no spiral arms and are labeled as S0, as their bulge is generally circular in shape.

In this scheme, spirals are classified as $\mathrm{Sa}, \mathrm{Sb}$ and $\mathrm{Sc}$, according to the tightness of the binding of their arms, with $\mathrm{Sa}$ being the the most tightly bound and Sc being most loosely bound. Barred spirals are accordingly labeled as SBa, SBb and SBc. Although earlier studies of spiral galaxy shapes have described these galaxies as a logarithmic spiral with constant pitch, a recent study [9] found this to be an inadequate discriminator for Hubble type spiral galaxies and consequently the correlation was poor. Hence, Ringermacher and Mead [9] have defined an elegant single parameter variable pitch formula for describing the spiral curve in terms of the polar co-ordinates $(\mathrm{r}, \Phi)$

$$
\mathrm{r}(\phi)=\mathrm{R}_{\Phi} /[1-\Phi \tan (\phi) \log (\phi / \Phi)]
$$

with $\Phi$ being the pitch at turnover, which has very good correlation with the Hubble classification, which are used as measuring function for spiral galaxies.

Following [11], the second set of measuring functions is defined on the variation of pixel intensities for the different structures of interest (SOI), like the bulge, disk and bar which have different col ours than the based on their composition of stars. So, for this measuring function, color segmentation is performed using an Otsu based algorithm and integer values of colors ranging from 1 to 7 based on the predominant color of the SOI (with violet(v) being 1 and red(r) being 7) are assigned. The size function $1(x, y)$ will thus be the sum of the two measuring functions. Thus

$$
\begin{gathered}
1(\mathrm{x}, \mathrm{y})=\text { shape measuring function }(\mathrm{m} . \mathrm{f} .)+ \\
\text { color } \mathrm{m} . \mathrm{f}
\end{gathered}
$$

in $(\mathrm{x}, \mathrm{y})$ space of the size function. Since elliptic and spiral shapes can be distinguished visually, they are treated separately. So E0-E7 are labeled as 1 to 7, respectively, and $\mathrm{Sa}, \mathrm{Sb}$ and $\mathrm{Sc}$, corresponding to $\Phi=0.4,0.5$ and 0.6, respectively, labeled as 1 to 3 .

Table 1. Computation of Size Functions

\begin{tabular}{|c|c|c|c|}
\hline $\begin{array}{c}\text { Galaxy } \\
\text { name }\end{array}$ & $\begin{array}{c}\text { Size } \\
\text { Function } \\
\mathbf{l}(\mathbf{x}, \mathbf{y})\end{array}$ & Classification & $\begin{array}{c}\text { Correctne } \\
\text { ss }\end{array}$ \\
\hline M89 & 7 & Elliptical & correct \\
\hline M32 & 9 & Elliptical & correct \\
\hline NGC4621 & 12 & Elliptical & correct \\
\hline NGC2273 & 48 & Barred spiral & correct \\
\hline NGC3198 & 68 & Barred spiral & correct \\
\hline NGC5754 & 54 & Barred spiral & correct \\
\hline NGC5010 & 16 & lenticular & correct
\end{tabular}

\section{RESULTS AND DISCUSSION}

Based on this classification we have applied our results to seven galaxies(Images in Figure 1), three elliptical, three spiral and one lenticular and have obtained size functions as defined in Table 1 and shown in the graph of Figure 2. The names of the galaxies, their image source, the calculated value of ellipticity or $\eta=10 \mathrm{x} \Phi$ from [10] and all color bands obtained from segmentation are listed.

From these results it may be inferred that assigning size functions can be an useful, simple and robust way of classifying galaxies. This is because the size functions for elliptical galaxies are lower than that for spirals and lenticulars, owing to the fact that ellipticals generally have one color in the red range, whereas spirals and lenticulars have several colors. In addition, The shape m.f. for ellipticals vary between 0 and 7 , whereas the integer values of shape m.f. of spirals range between 30 and 60 . Thus, ellipticals have the lowest size functions ranging from 7 to 14 , lenticulars have intermediate values and spirals have the largest values. This method is simpler to use than the soft computing based methods as integer values of size functions are indicators of the class the galaxy belongs to. This method is also robust as they rely only on these integer valued functions. Thus these features of size function based classification render it attractive for automated classification.

Future work would be aimed at classifying many more galaxies and standardizing the limits of the values of the size functions for the different categories, so that the procedure can be automated on the computer. 


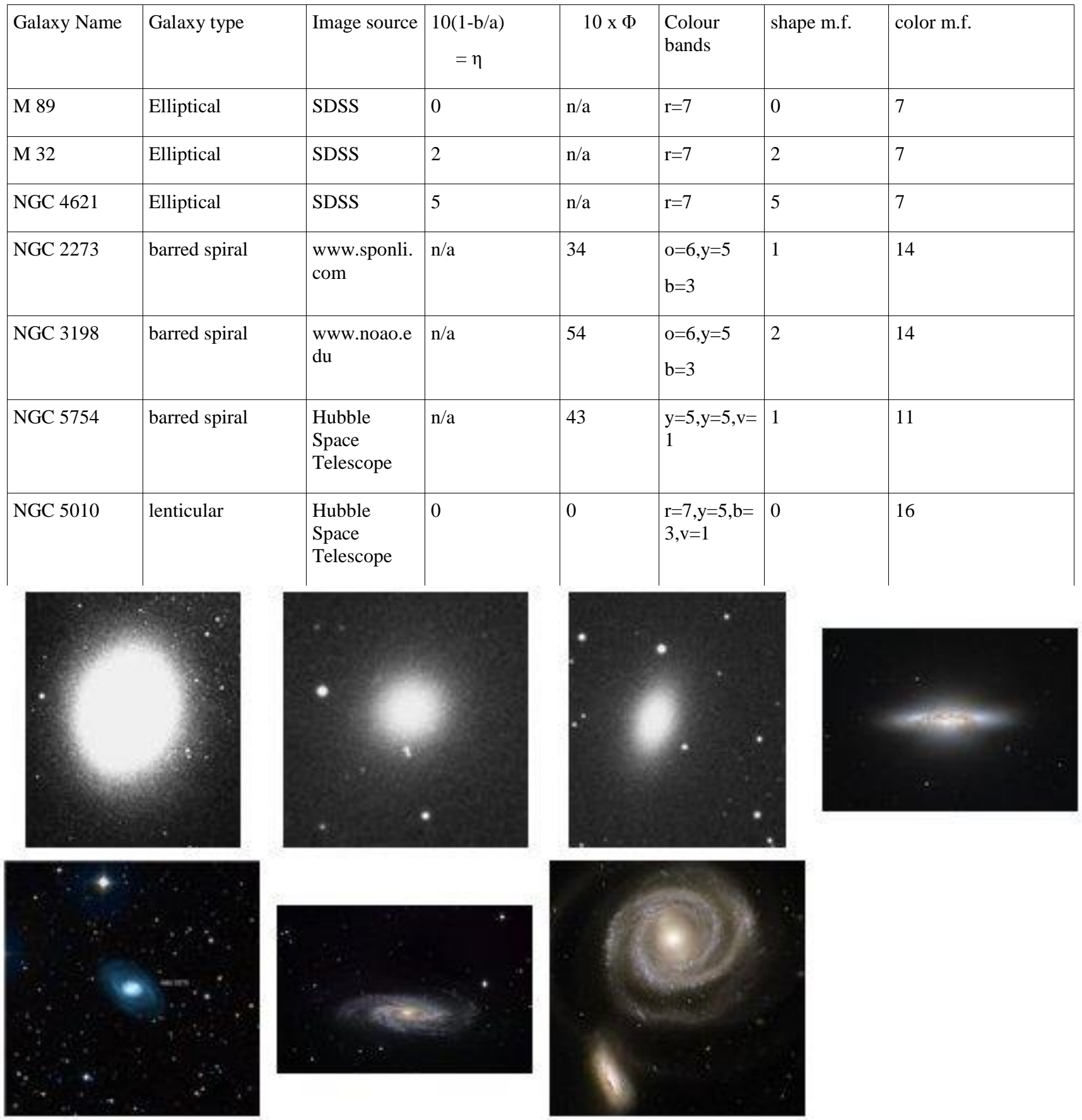

Fig 1: Galaxy images from top left clockwise: (a) M89 (b) M32 (c) NGC 421 (d) NGC 5010 (e) NGC 2273 (f) NGC 3198 (g) NGC 5754. The particulars of the galaxies are given in the accompanying table above.

\section{ACKNOWLEDGMENTS}

The author wishes to acknowledge a major research project (MRP) grant from University Grants Commission (F: 37-534 ('09)), Government of India.

\section{REFERENCES}

[1] Hubble, E. P. (1926). Extragalactic nebulae. Astrophysics Journal, 64, 321-369.

[2] de Vaucouleurs, G. 1959, Handbuch der Physik, 53, 275.
[3] Sandage, A. 1961, The Hubble Atlas of Galaxies, Carnegie Inst. of Wash. Publ. No. 618; Sandage, A. 1975, in Galaxies and the Universe, Stars and Stellar Systems, Vol. IX, A.

[4] Frei Z. Automatic Morphological Classification of Galaxies, Astrophysics and Space Science, 1999, 269270, 577-583.

[5] Kent, S. M. 1985, Bulge/disk decompositions, Astrophys.J.Suppl. 59, 115-159. 
[6] Okamura S. Kodaira K., Watanabe M, 1984, Digital Surface Photometry of Galaxies Toward a Parameter Representing the Luminosity Distribution, Astrophys. J. 280. 7-14.

[7] Calleja J. de la, Fuentes O., Automated Classification of Galaxy Images, Knowledge Based Intelligent and Information and Engineering Systems, 2004, Lecture Notes In Computer Science (LNCS) 3215, 411-418.

[8] Frosini, P. Measuring shapes by size functions, 1991, Proceedings of SPIE on Intelligent Robots and Computer Vision X, Boston Mass. 1607, 122-133.
[9] Verri, A., Uras C., Frosini, P., and Ferri, M. (1993) On the use of size functions for shape analysis, Biol. Cybernetics, 70, 99-107.

[10] Ringermacher H. I., Mead L. R., 2009, A New Formula Describing the Scaffold Structure of Spiral Galaxies, Mon. Not. Roy. Astrom. Soc., 397, 164-171.

[11]Ferri M., Lombardi S. and Palloti C., Leukocyte classification by size function, 1994, Proceed. 2nd workshop on applications of Computer Vision, 223-229.

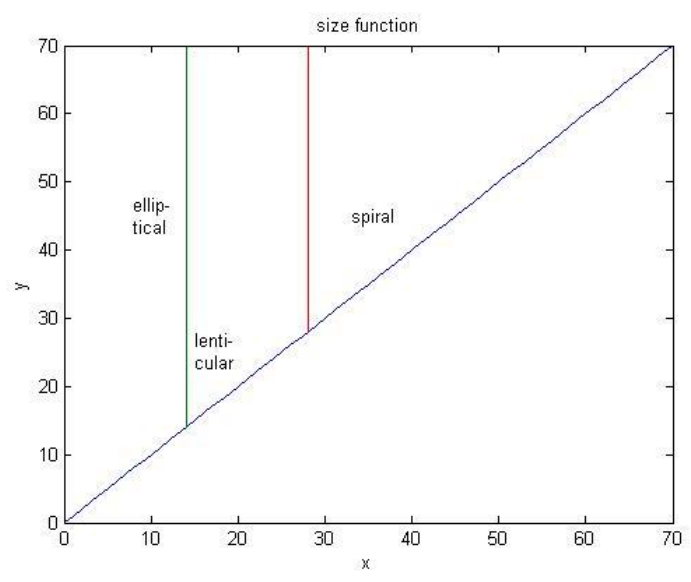

Fig 2: Size Functions 